\title{
Measuring and Mapping Cardiac Fiber and Laminar Architecture Using Diffusion Tensor MR Imaging
}

\author{
Patrick Helm ${ }^{1}$, Mirza Faisal Beg${ }^{2}$, Michael I. Miller ${ }^{2}$ and Raimond L. Winslow ${ }^{1}$ \\ The Center for Cardiovascular Bioinformatics \& Modeling ${ }^{1}$ and The Center for Imaging \\ Sciences ${ }^{2}$, The Johns Hopkins University School of Medicine and Whiting School of \\ Engineering, Baltimore MD 21218 USA
}

\begin{abstract}
The ventricular myocardium is known to exhibit a complex spatial organization, with fiber orientation varying as a function of transmural location. It is now well established that diffusion tensor magnetic resonance imaging (DTMRI) may be used to measure this fiber orientation at high spatial resolution. Cardiac fibers are also known to be organized in sheets with surface orientation varying throughout the ventricles. In this paper, we review results on use of DTMRI for measuring ventricular fiber orientation, as well as present new results providing strong evidence that the tertiary eigenvector of the diffusion tensor is aligned locally with the cardiac sheet surface normal. Considered together, these data indicate that DTMRI may be used to reconstruct both ventricular fiber and sheet organization. We also present the Large Deformation Diffeomorphic Metric Mapping (LDDMM) algorithm and show that this algorithm may be used to bring ensembles of imaged and reconstructed hearts into correspondence (e.g., registration) so that variability of ventricular geometry, fiber and sheet orientation may be quantified. Ventricular geometry and fiber structure is known to be remodeled in a range of disease processes $^{2-4}$, however, descriptions of this remodeling have remained subjective and qualitative. We anticipate that use of DTMRI for reconstruction of ventricular anatomy coupled with application of the LDDMM method for image volume registration will enable the detection and quantification of changes in cardiac anatomy that are characteristic of specific disease processes in the heart.
\end{abstract}




\section{INTRODUCTION}

The fiber structure of the cardiac ventricles plays a critical role in determining properties of electrical conduction in the heart. Conduction is anisotropic, with current spread being most rapid in the direction of the fiber long axis ${ }^{5,6}$. Conduction is also influenced by properties of tissue geometry such as expansion and contraction ${ }^{7-9}$, as well as by the spatial rate of change of fiber orientation ${ }^{10}$. Remodeling of ventricular geometry and fiber organization is a prominent feature of several cardiac pathologies. These alterations may figure importantly in arrhythmogenesis ${ }^{11}$. A detailed knowledge of ventricular fiber structure, how it may be remodeled in cardiac pathology, and the effects of this remodeling on ventricular conduction is therefore of fundamental importance to the understanding of cardiac electro-mechanics in health and disease.

Ventricular fiber orientation was first studied by means of fiber dissections ${ }^{12-14}$ and histological measurements in transmural plugs of ventricular tissue ${ }^{15-19}$. The principle conclusions of this work were that cardiac fibers are arranged as counter-wound helices encircling the ventricular cavities and that fiber orientation is a function of transmural location, with fiber direction being oriented predominantly in the base-apex direction on the epicardial and endocardial surfaces and rotating to a circumferential direction in the midwall. These initial fiber dissection studies were restricted to a small number of myocardial measurement sites. Nielsen et $\mathrm{al}^{20}$ overcame this limitation by using a custom-built histological apparatus to measure inclination angle at up to 14,000 points throughout the myocardium, with a resolution of $\sim 500,2500$ and $5000 \mu \mathrm{m}$ in the radial, longitudinal and circumferential directions, respectively. This work led to the first complete reconstruction of ventricular geometry and fiber orientation - an achievement which has had significant impact on the cardiac mechanics and electrophysiology communities.

Despite this important advance, histological methods continue to suffer from the disadvantage that they require many weeks to even months for reconstruction of a single heart. As a consequence, only a relatively small numbers of hearts have been reconstructed. Full reconstruction of ventricular fiber structure in diseased hearts has never been performed. Because of the long reconstruction times involved, it will not be possible to use current histological methods to acquire data sets containing many instances of reconstructed normal and pathological hearts. Methods for registration of normal and diseased hearts so that consistent changes of fiber structure may be assessed in corresponding regions have not been developed and there have been few efforts to relate effects of disease-induced remodeling of ventricular fiber structure to properties of electrical conduction ${ }^{21}$. These problems must be solved if we are to identify quantitatively the spatial properties of ventricular remodeling of fiber structure and relate this remodeling to changes in properties of ventricular conduction.

Recently, diffusion tensor magnetic resonance imaging (DTMRI), a technique for measuring the self-diffusion of protons in fibrous tissue, has emerged as a powerful new tool for the rapid measurement of cardiac geometry and fiber structure at high spatial resolution. In this paper we will summarize the current state of DTMRI for use in reconstruction of ventricular anatomy. We first review results regarding the application of DTMRI to measure fiber orientation in the cardiac ventricles. These results demonstrate that the primary eigenvector of the diffusion tensor measured at each image voxel is aligned with the direction of the cardiac fiber long-axis and that both ventricular geometry and fiber orientation may be measured rapidly and at high spatial resolution using DTMRI. Second, we present new data providing strong support for the hypothesis 
that the tertiary eigenvector of the diffusion tensor measured at each image voxel is aligned with the surface normal to the cardiac sheets ${ }^{22}$. Finally, we describe a quantitative mathematical approach known as the large deformation diffeomorphic metric mapping (LDDMM) method by which ensembles of imaged and reconstructed hearts may be registered so that statistical analysis of variation of ventricular geometry and shape may be performed.

\section{METHODOLOGY}

\section{DTMRI Imaging Methods}

DTMRI methods have been described previously ${ }^{23,24}$. Anesthesia was induced with sodium thiopental and maintained after intubation with isofurane. A mid-line thoracotomy was performed, animals were heparinized and cardiac arrest was induced with a bolus of potassium chloride. Following explantation, a Langendorff preparation was used to perfuse the heart with saline solution until coronary vessels were clear. Vinyl Polysiloxane was injected into each of the four chambers via the tri-cuspid and mitral valves in order to maintain diastolic conformation during imaging. The hearts were then perfused with and stored in a 7\% by volume isotonic solution of formaldehyde. Care was taken to fix hearts in the same cardiac phase so that their fiber orientations would be similar. Small variations in cardiac phase during fixation are not problematic since fiber angles do not change significantly between diastole and systole ${ }^{15}$. Each heart was placed in an acrylic container filled with the perfluoropolyether Fomblin (the low dielectric effect and minimal MR signal of Fomblin increases contrast and eliminates unwanted susceptibility artifacts near the boundaries of the heart). The long axis of each heart was aligned with the z-axis of the scanner and images were acquired with a 4-element phased array coil on a $1.5 \mathrm{~T}$ GE CV/I MRI Scanner. The system had a $40 \mathrm{mT} / \mathrm{m}$ maximum gradient amplitude and a $150 \mathrm{~T} / \mathrm{m} / \mathrm{s}$ slew rate. Hearts were placed in the center of the coil and a 3-D fast spin-echo sequence (3DFSE) was used to acquire diffusion images ${ }^{24}$. Total scan time was approximately 60 hours per heart.

\section{Reconstruction of Ventricular Geometry and Fiber Organization}

After imaging, the three principle eigenvalues and eigenvectors were computed for each voxel from the diffusion tensors ${ }^{22,}{ }^{25}$. Voxels in the 3D DTMRI images representing compact myocardium were identified using a parametric active contouring method implemented as part of HeartWorks ${ }^{26}$. Ventricular geometry and fiber orientation were modeled using three-dimensional finite elements as described by Nielson et al. ${ }^{20}$ using a graphical user interface implemented in Matlab. Diffusion tensor eigenvectors corresponding to voxels within the finite element boundaries were used to study the fiber structure of the ventricles. These eigenvectors were transformed into local material coordinates of the model as described by LeGrice et al. ${ }^{27}$. Fiber inclination angle $\theta$ (the angle formed by the circumferential epicardial tangent vector $\mathbf{t}_{\mathbf{1}}$ and the projection of the primary eigenvector onto the surface tangent plane defined by vectors $\mathbf{t}_{\mathbf{1}}$ and the axial tangent vector $\mathbf{t}_{2}$ ) was used as a measure of fiber orientation and was computed as shown in Fig. 1. The circumferential tangent vector $\mathbf{t}_{\mathbf{1}}$ was constrained to the $\mathrm{xy}$ plane. Intersection angle $\phi$ was used as a measure of cardiac sheet orientation and was defined as the angle formed by the surface normal and the projection of the tertiary eigenvector onto the plane defined by the surface normal $\mathbf{n}$ and $\mathbf{t}_{2}$. A total of 11 normal and 7 
tachycardia pacing-induced failing canine hearts were reconstructed using these methods. Data was loaded into the Cardiac Anatomic Database System (CADS) ${ }^{28}$ for visualization and analysis.

\section{Anatomical Registration and Structural Analysis of Imaged Hearts}

We have recently developed a mathematical method known as the LDDMM algorithm for accurate registration of ensembles of imaged and reconstructed heart anatomies ${ }^{23,29}$. In this method, an anatomy is defined as collection

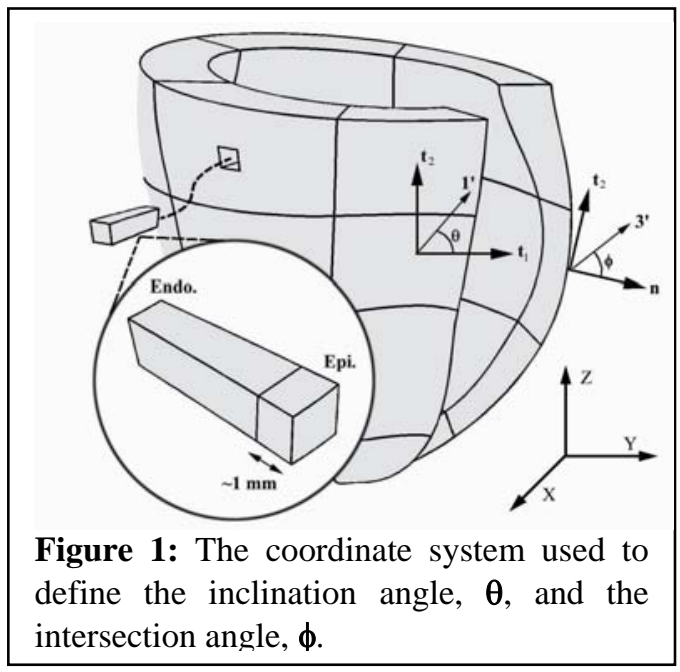
of landmarks, curves, surfaces, sub-volumes and tensors. A "template" anatomy is selected from a database of imaged hearts and large deformation mappings are generated and used to deform this template onto "target" anatomies (e.g., other imaged hearts in the database). The transformations are constrained to be $1: 1$ and differentiable with differentiable inverse so that every point in the template has a corresponding point in the target, connected sets of template image points remain connected in the target, surfaces are mapped as surfaces and volumes are mapped as volumes, thereby maintaining the global relationships between these structures. Regional anatomic variability is then studied as a property of the local variability of these transformations. The advantages of the LDDMM method over small-deformation mapping schemes are that the computed transformations are invertible and therefore the choice of an anatomic template is arbitrary and LDDMM computes a geodesic which is a shortest length path in the space of transformations matching the given imagery, thereby quantifying deformation via a scalar metric distance.

\section{RESULTS}

Substantial data now confirms the hypothesis that the primary eigenvector of the diffusion tensor is aligned locally with the long-axis of cardiac fibers ${ }^{22,24,26,30,31}$. As an example, Fig. 2A shows fiber inclination angle (ordinate) as a function of transmural depth (abscissa). Open symbols show histological measurements from transmural tissue plugs and filled circles show DTMRI estimates obtained at the same locations of these transmural tissue plugs. Histological and DTMRI-based measurements are in close agreement. We have also shown that DTMRI can be used to image and reconstruct fiber orientation throughout the cardiac ventricles ${ }^{23}{ }^{26}$. Figure 2B shows an example of reconstruction of both ventricular geometry and fiber orientation in a normal canine heart. Fiber inclination angle is color-coded, with blue indicating the circumferential direction and red indicating the base-apex direction. Imaging resolution is $\sim 300 \mu \mathrm{m}$ inplane and $\sim 800 \mu \mathrm{m}$ out-of-plane. Imaging at this resolution enables measurement of fiber orientation at roughly two orders of magnitude more points than is possible with histological reconstruction methods ${ }^{20}$. Figure 2C shows that fiber orientation may be reconstructed accurately even in regions of the ventricles where fiber curvature is large (e.g., the apex of the heart). Finally, Fig. 2D shows that fiber-tracing methods may be used to map fiber trajectories within the heart in a matter reminiscent of the original fiber 

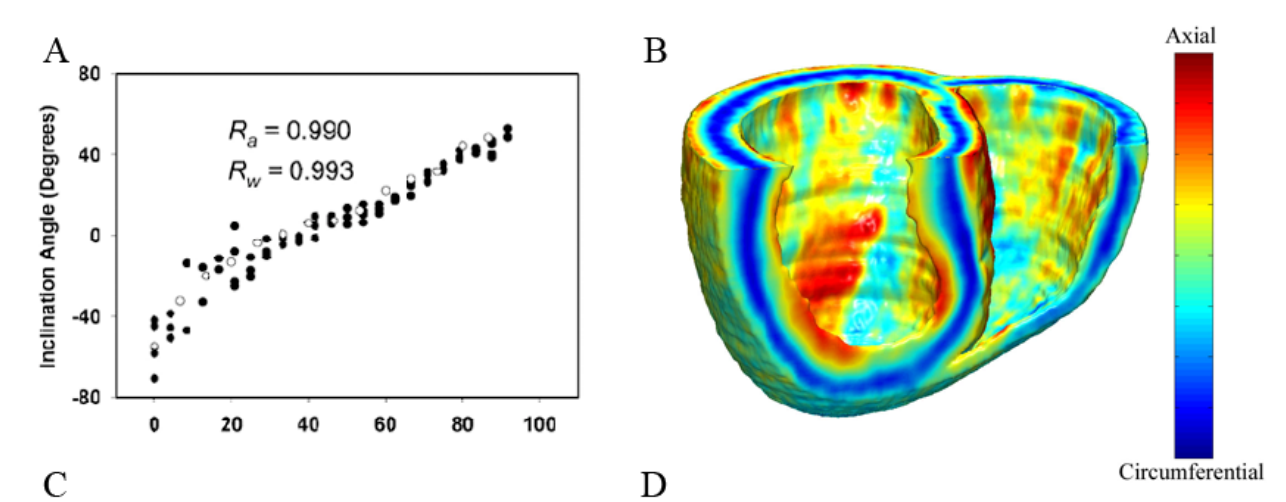

$\mathrm{C}$

$\mathrm{D}$
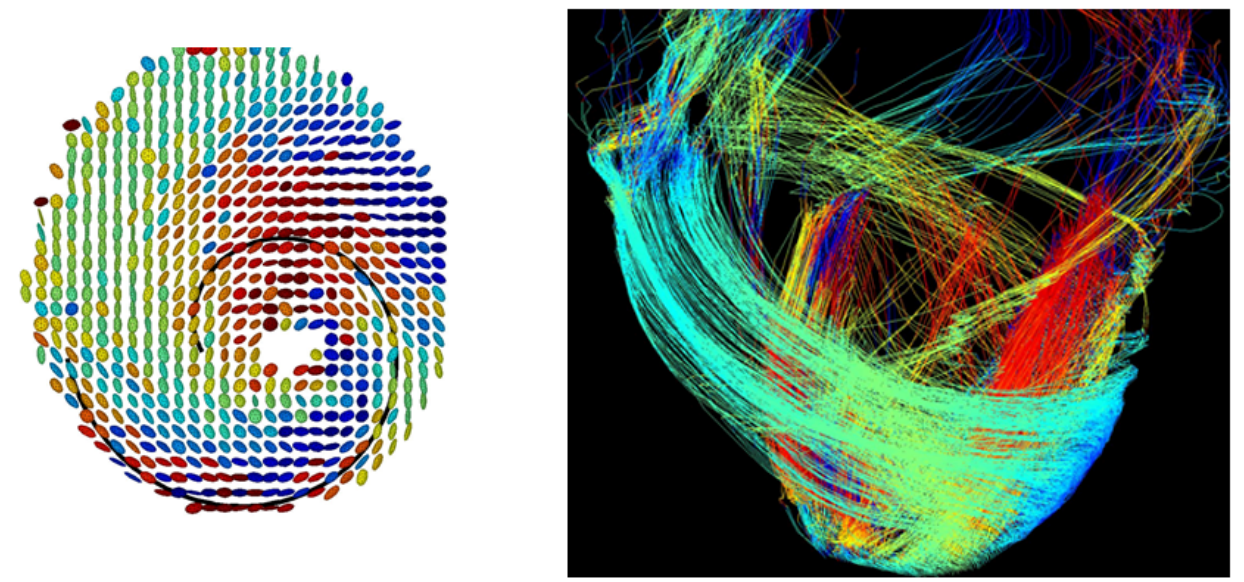

Figure 2: A) Comparison of histological and DTMRI-bases estimates of fiber inclination angle at corresponding transmural location. B) Reconstruction of fiber inclination angle throughout the canine cardiac ventricles. C) Map of fiber inclination angle at the apex of a canine heart. D) fiber tracing verformed in a canine heart using DTMRI data.

dissections of McCallum, Mall and Fox ${ }^{12-14}$.

Cardiac fibers are known to be organized in sheets approximately four cells in thickness and which radiate outward from the endocardial to the epicardial surface ${ }^{1,27}$. Local surface orientation of these sheets varies spatially. In previous work, we compared the intersection angle obtained by projection of the tertiary eigenvector of the diffusion tensor onto a plane defined by the epicardial and axial tangent vectors (see Fig. 1) with the intersection angle generated by projection of surface normal to the cardiac sheets measured histologically by Nielsen et $\mathrm{al}^{20}$. These data are shown in Figs. 3A-C. Figure 3A shows color-coded DTMRI-based estimates of this inclination angle in a single shortaxis section of the rabbit heart and Fig. 3B shows the data of Fig. 3A smoothed using a median filter ${ }^{22}$. Figure 3C shows interpolated intersection angles of the surface normal to the ventricular sheets determined by LeGrice et al in canine heart ${ }^{1,27}$. The short axis section of Fig. 3C was selected to be positioned at roughly the same percentage distance from base to apex as was the section of Figs. 3A and B. The qualitative correlation between intersection angles of the tertiary eigenvector and the sheet normals is striking, despite being from not only different hearts, but also different species.

More recent theoretical analyses coupled with histological reconstructions have predicted and verified the existence of two populations of sheets differing in orientation by approximately $90^{\circ}$ in the canine ${ }^{32}$ and ovine ${ }^{33}$ ventricles. We therefore sought to 
determine if DTMRI may be used to reconstruct these two differing populations of sheet angles $^{24}$. To do this, we measured intersection angles for the tertiary eigenvector of the diffusion tensor at ventricular locations similar to those where previous histological measurements of dual sheet structure have been reported ${ }^{32,33}$. In these prior experimental studies, left ventricular transmural blocks of tissue were isolated and sectioned in $\sim 1 \mathrm{~mm}$ increments parallel to the epicardial tangent plane and measurements of intersection angles were averaged within these regions. Using a similar approach, we isolated 24 transmural regions by selecting four transmural regions each from within the anterior base, lateral base, posterior base, anterior apex, lateral apex, and posterior apex of the left ventricle. These 24 regions were divided into $\sim 1 \mathrm{~mm}$ cube sub-regions each containing on average 291 tensor measurements. Based on normalized transmural depth, we assigned each cube as being in the epicardium (0\%-20\%), sub-epicardium (20\%-40\%), mid-wall (40\%-60\%), sub-endocardium (60\%-80\%) or endocardium (80\%-100\%).

Figures 3D-E show analyses of the distribution of intersection angles computed from the tertiary eigenvector in the four transmural regions located in the apical-anterior LV free wall for two hearts (gray scales). We observed that within each region of each heart, distribution of intersection angle exhibited a single dominant peak (Fig. 3D). The dominant intersection angle differed at various anatomic locations. This finding is similar to observations made using histological reconstruction methods in which each report indicates the presence of a dominant muscle layer ${ }^{32,33}$. Dominant populations were located on average at approximately $45^{\circ}$ and $117^{\circ}$. Histological measurements have

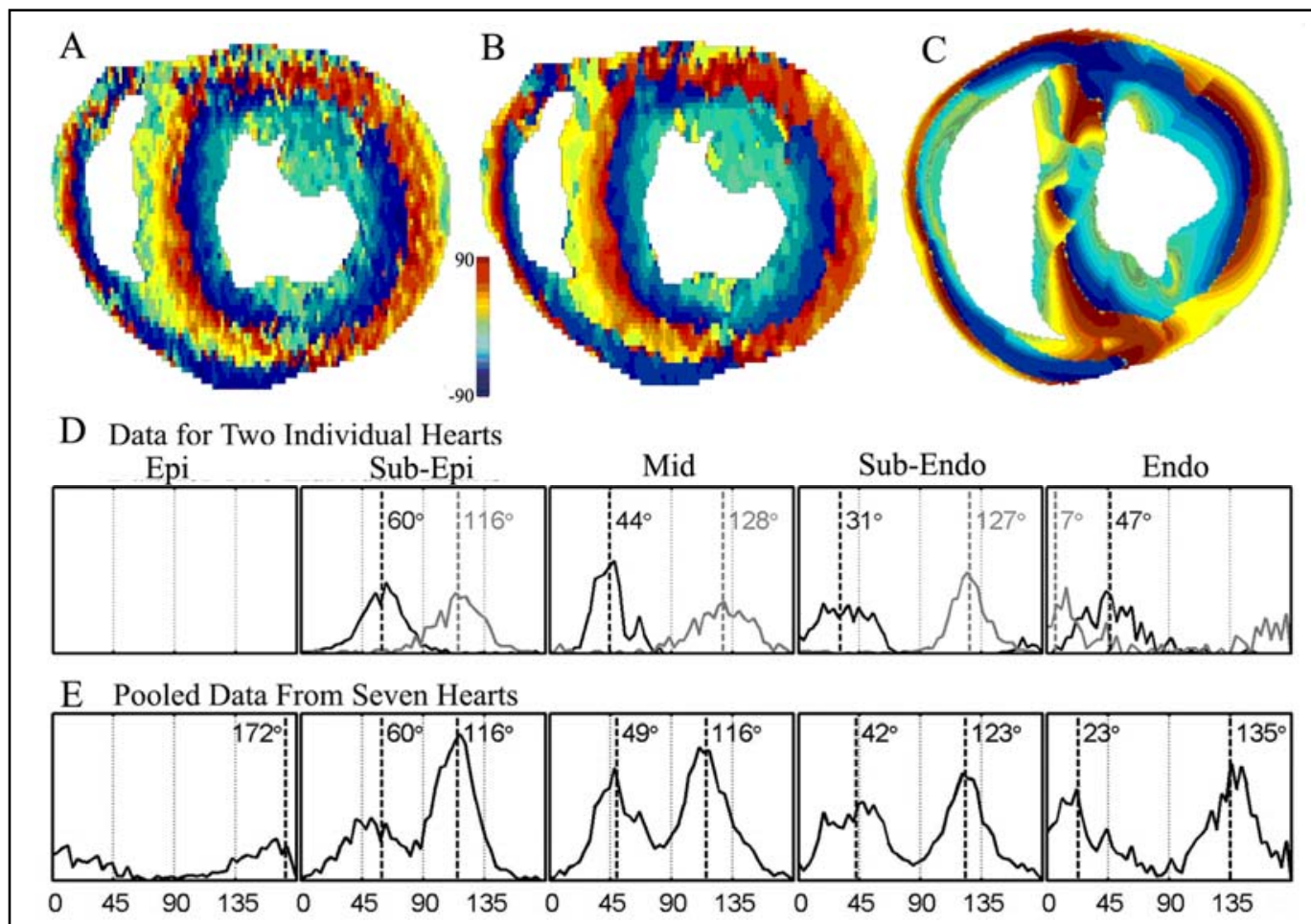

Figure 3: A) Intersection angle measured using DTMRI in a short-axis section of the rabbit ventricle. B) Section in panel A smoothed using a median filter. C) Intersection angle measured by LeGrice et $\mathrm{al}^{1}$ in a short axis section of the canine heart. D) Histograms of intersection angle measured in the indicated regions of canine myocardium for two different hearts (grey vs black lines). E) Pooled data, as in D, from 7 normal canine hearts. 


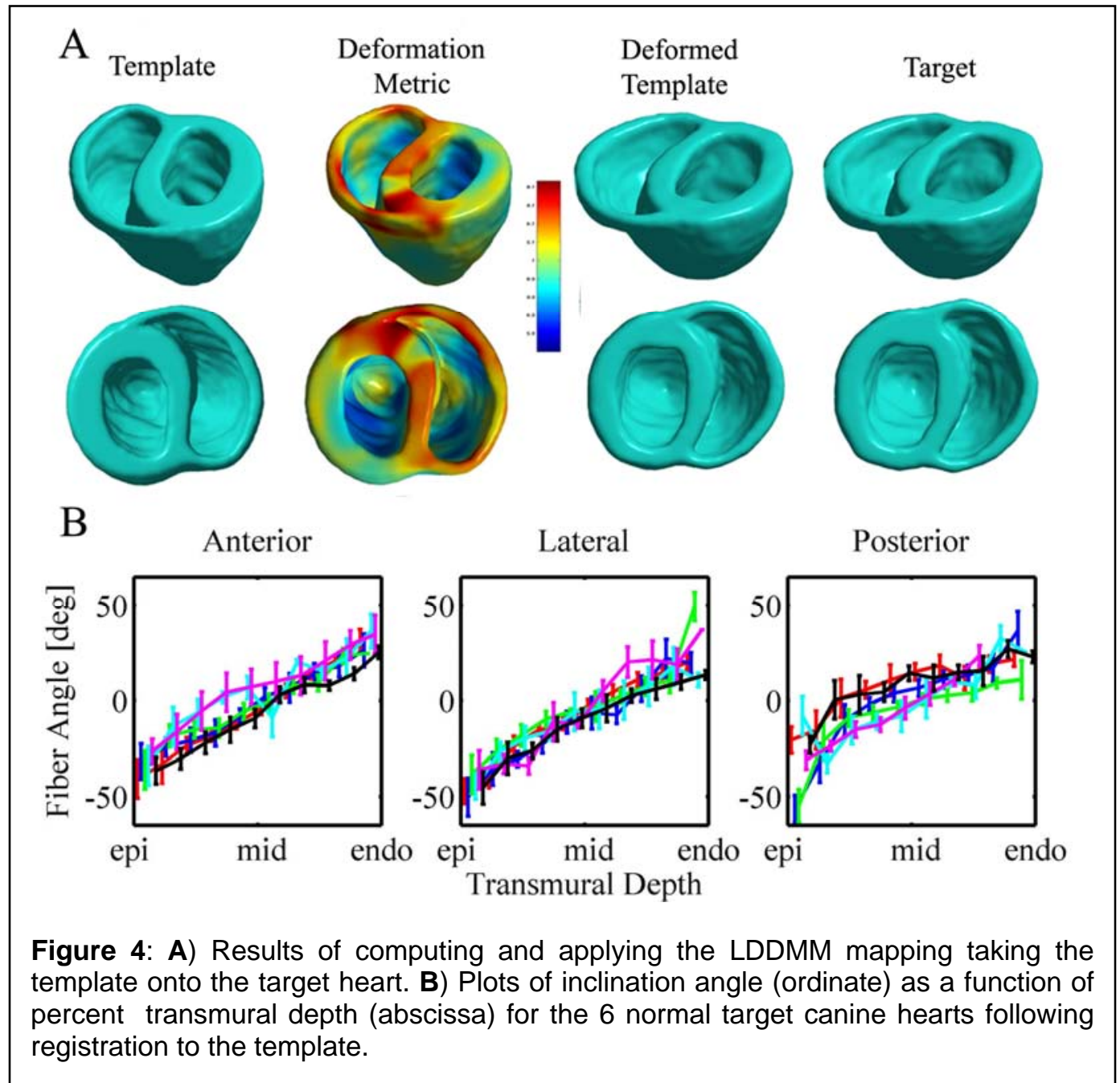

indicated similar values for sheet angles. Specifically, Dokos et al. identified a dominant mid-wall muscle layer at $\sim 45^{\circ}$ and another at $\sim 110^{\circ 34}$ and Ashikaga et al. observed angles at $36^{\circ}$ and another at $\sim 70-90^{\circ} 35$. Finally, when the distribution angles for all hearts were pooled, a bi-modal distribution of angles per location emerged (Fig. 3E), with the two dominant angles being equally likely. This is similar to the results of Arts et al. in which pooled histological measurements were obtained from six canine hearts. Peaks of pooled intersection angles were observed at $45^{\circ}$ and $135^{\circ}$ per location with similar likelihood $^{32}$. These data provide strong evidence that the tertiary eigenvector of the diffusion tensor corresponds to the surface normal of the laminar sheets.

The data presented above demonstrate how DTMRI may be used to reconstruct the detailed anatomic structure of the cardiac ventricles. Using this approach, we have imaged and reconstructed a population of 11 normal and 7 failing canine hearts (failure was induced using the tachycardia pacing procedure). Our goal is to first assess withinclass variability of these anatomies (normal vs normal, failing versus failing) and then, using estimates of within-class variability, extend the analyses to identify between class differences of both geometry, fiber and sheet orientation between normal versus failing hearts. 
Figure 4 shows results of applying the LDDMM algorithm to register a template to a target heart. In Fig. 4C the left column labeled "template heart" present two views of the structure of a normal canine heart selected as the template. The right column labeled "target" presents two views of a failing canine heart selected as the "target". The column labeled "deformation metric" shows a color-coded representation of the displacements needed to bring every point in the template into 1:1 correspondence with a point in the target heart. The column labeled "deformed template" shows the results of deforming the template onto the target heart. Comparison of the two right-most columns shows that the mapping computed using the LDDMM algorithm leads to accurate registration of target and template hearts.

In Fig. 4D we show fiber inclination of 6 normal canine hearts (colors) taken at corresponding transmural locations in the anterior, lateral, and posterior wall of the left ventricle. Locations were selected by choosing a transmural set of voxels in the anterior, lateral, and posterior left ventricular walls of the normal template heart and then using the LDDMM mapping to locate corresponding sets of voxels in each of the six normal target hearts. The ordinate plots average fiber inclination angle at each transmural location (abscissa) for each of the target hearts. Error bars indicate the standard deviation of inclination angle calculated per heart at each transmural location. This variation reflects both anatomical variability as well as measurement noise. The mean standard deviation calculated over all transmural locations and hearts is 6.0, 5.9 and 5.7 degrees for anterior, lateral and posterior LV locations, respectively. The standard deviation of mean inclination angle calculated over all target hearts and transmural locations is 3.3, 4.7 and 7.2 degrees for anterior, lateral and posterior LV locations, respectively. These data therefore offer an example of how the LDDMM algorithm may be used to register hearts in order to assess anatomic variability at corresponding locations. In particular, these data show indicate that variation of fiber inclination angle at corresponding points over an ensemble of hearts is surprisingly small.

\section{SUMMARY}

It is now clear that the diffusion tensor primary eigenvector is aligned locally with the cardiac fiber long-axis and therefore DTMRI may be used for rapid measurement of fiber orientation throughout the canine ventricles at high spatial resolution. The data of Fig. 3 showing that intersection angles measured using DTMRI agree closely with those measured using histological reconstruction provide strong evidence supporting the hypothesis that the diffusion tensor tertiary eigenvector is aligned locally with the cardiac sheet surface normal. Considered together, these observations suggest that DTMRI may be used to reconstruct both ventricular fiber and sheet organization. Finally, we show that the LDDMM algorithm may be used to register imaged and reconstructed hearts so that variability of ventricular geometry, fiber and sheet orientation may be quantified over ensembles of imaged hearts. Ventricular geometry and fiber structure is known to be remodeled in a range of disease processes ${ }^{2-4}$, however, descriptions of this remodeling have remained subjective and qualitative. Future research will combine use of DTMRI with the LDDMM method to detect statistically significant changes of cardiac anatomy that are characteristic of specific disease processes in the heart.

\section{ACKNOWLEDGEMENTS}


Work supported by NIH HL70894, HL52307, the Whitaker Foundation, the Falk Medical Trust and IBM Corporation. All DTMRI datasets employed in this study and the Cardiac Anatomic Database System are downloadable from http://www.ccbm.jhu.edu.

\section{REFERENCES}

1. LeGrice, I. J., et al. 1995. Laminar structure of the heart: ventricular myocyte arrangement and connective tissue architecture in the dog. Am. J. Physiol. 269(2Pt2): H571-H582.

2. Maron, B. J., et al. 1979. Quantitative analysis of cardiac muscle cell disorganization in the ventricular septum. Comparison of fetuses and infants with and without congenital heart disease and patients with hypertrophic cardiomyopathy. Circulation. 60: 685-96.

3. Maron, B. J., T. J. Anan \& W. C. Roberts. 1981. Quantitative analysis of the distribution of cardiac muscle cell disorganization in the left ventricular wall of patients with hypertrophic cardiomyopathy. Circulation. 63: 882-94.

4. Roberts, W. C., R. J. Siegel \& B. M. McManus. 1987. Idiopathic Dilated Cardiomyopathy: Analysis of 152 necropsy patients. Am J Cardiol. 60: 1340-1355.

5. Franzone, P. C., et al. 1998. Spread of excitation in 3-D models of the anisotropic cardiac tissue. II. Effects of fiber architecture and ventricular geometry. Math Biosci. 147: 131-171.

6. Kanai, A. \& G. Salama. 1995. Optical mapping reveals that repolarization spreads anisotropically and is guided by fiber orientation in guinea pig hearts. Circ Res. 77: 784-802.

7. Rohr, S. \& B. M. Salzberg. 1994. Characterization of impulse propagation at the microscopic level across geometrically defined expansions of excitable tissue: multiple site optical recording of transmembrane voltage (MSORTV) in patterned growth heart cell cultures. J Gen Physiol. 104: 287-309.

8. Rohr, S., A. G. Kleber \& J. P. Kucera. 1999. Optical recording of impulse propagation in designer cultures. Cardiac tissue architectures inducing ultra-slow conduction. Trends Cardiovasc Med. 9: 173-9.

9. Kucera, J. P., A. G. Kleber \& S. Rohr. 1998. Slow conduction in cardiac tissue, II: effects of branching tissue geometry. Circ Res. 83: 795-805.

10. Taccardi, B., et al. 1994. Effect of myocardial fiber direction on epicardial potentials. Circulation. 90: 3076-90.

11. Chen, P. S., et al. 1993. Effects of myocardial fiber orientation on the electrical induction of ventricular fibrillation. American Journal of Physiology. 264: H1760-73.

12. MacCallum, J. B. 1900. On the muscular architecture and growth of the ventricles of the heart. Johns Hopkins Hosp. Rep. 9: 307-335.

13. Mall, F. 1911. On the muscular architecture of the ventricles of the human heart. American Journal of Anatomy. 11: 211-266.

14. Fox, C. C. \& G. M. Hutchins. 1972. The architecture of the human ventricular myocardium. Johns Hopkins Med. J. 25: 289-299.

15. Streeter, D., et al. 1969. Fiber orientation in the canine left ventricle during diastole and systole. Circulation Research. 24: 339-347.

16. Streeter, D. D. \& W. T. Hanna. 1973. Engineering mechanics for successive states in canine left ventricular myocardium. II. Fiber angle and sarcomere length. Circ Res. 33: 656-664.

17. Ross, A. A. \& D. D. Streeter, Jr. 1979. Myocardial fiber disarray [letter]. Circulation. 60: 1425-6.

18. Streeter, D. 1979. Chapter 4. Gross morphology and fiber geometry of the heart. In Handbook of Physiology, The Cardiovascular System I. Berne, R., Ed.: 61-112. American Physiological Society. Bethesda, MD.

19. Streeter, D. D. \& C. Ramon. 1983. Muscle pathway geometry in the heart wall. Journal of Biomechanical Engineering. 105: 367-73.

20. Nielsen, P. M., et al. 1991. Mathematical model of geometry and fibrous structure of the heart. American Journal of Physiology. 260: H1365-78. 
21. Anderson, K. P., et al. 1993. Myocardial Electrical Propagation in Patients with Idiopathic Dilated Cardiomyopathy. J. Clin. Invest. 92: 122-140.

22. Scollan, D., et al. 1998. Histologic validation of reconstructed myocardial microstructure from high resolution MR diffusion tensor imaging. Am. J. Physiol. 275 (Heart Circ. Physiol. 44): H2308-H2318.

23. Beg, F. M., et al. 2004. Computational Cardiac Anatomy Using Magnetic Resonance Imaging. Mag. Res. Med. 52: 1167-1174.

24. Helm, P. A., et al. 2004. 3D diffusion tensor imaging and quantification of cardiac laminar structure. MRM. submitted.

25. Basser, P., J. Mattiello \& D. LeBihan. 1994. Estimation of the effective self-diffusion tensor from the NMR spin echo. J Magn Reson B. 103: 247-54.

26. Scollan, D., et al. 2000. Reconstruction of cardiac ventricular geometry and fiber orientation using GRASS and diffusion-tensor magnetic resonance imaging. Annals BME. 28: 934-944.

27. Legrice, I. J., P. J. Hunter \& B. H. Smaill. 1997. Laminar structure of the heart: a mathematical model. Am J Physiol. 272: H2466-76.

28. Winslow, R., et al. 2002. Imaging-Based Integrative Models of the Heart: Closing the Loop Between Experiment and Simulation. Novartis Foundation Symposium 247 on In Silico Simulation of Biological Processes129-140.

29. Cao, Y., et al. 2004. Large deformation metric mapping of vectior fields. IEEE Trans Biomed. Imag. submitted.

30. Hsu, E. W., et al. 1998. Magnetic resonance myocardial fiber-orientation mapping with direct histological correlation. Am J Physiol. 274: H1627-H1634.

31. Holmes, A., D. Scollan \& R. Winslow. 1999. Direct histological validation of diffusion tensor MRI in formalin-fixed myocardium. Mag. Res. Med. 44: 157-160.

32. Arts, T., et al. 2001. Relating myocardial laminar architecture to shear strain and muscle fiber orientation. Am J Physiol Heart Circ Physiol. 280: H2222-9.

33. Harrington, K. B., et al. 2004. Direct measurement of transmural laminar architecture in the anterolateral wall of the ovine left ventricle: New implications for wall thickening mechanics. Am J Physiol Heart Circ Physiol00813.2004.

34. Dokos, S., et al. 2002. Shear properties of passive ventricular myocardium. Am J Physiol Heart Circ Physiol. 283: H2650-9.

35. Ashikaga, H., et al. 2004. Transmural left ventricular mechanics underlying torsional recoil during relaxation. Am J Physiol Heart Circ Physiol. 286: H640-7. 